\title{
Classical theory of two-dimensional time-domain terahertz spectroscopy
}

\author{
Toshiaki Hattoria) \\ Institute of Applied Physics, University of Tsukuba, 1-1-1 Tennodai, Tsukuba, Ibaraki 305-8573, Japan
}

(Received 25 August 2010; accepted 11 October 2010; published online 24 November 2010)

\begin{abstract}
A general theoretical framework of two-dimensional time-domain second-order and third-order terahertz spectroscopy has been presented. The theoretical treatment is based on a classical and phenomenological model with weak nonlinearities. Three types of nonlinearity sources, anharmonicity, nonlinear coupling, and nonlinear damping, were considered. The second-order $\mathrm{THz}$ spectroscopy has an exact correspondence to fifth-order off-resonance Raman spectroscopy, and it has been shown that the present treatment gives exactly the same results as of the quantum mechanical theory under the weak nonlinearity condition. General expressions for the nonlinear signal have been obtained for a single-mode system, and numerical calculations for delta-function incident terahertz pulses were shown. For the third-order signal, two-level systems were also considered for comparison. Contributions of two types of incident pulse sequences have been studied separately in the third-order signals. Profiles of the two-dimensional signals were found to depend on the origin and order of the nonlinearity and also on the pulse sequence. The results of the present study show that the two-dimensional signal features of second- and third-order nonlinear terahertz spectroscopy can clarify the nature of the system which is not accessible using linear spectroscopy. (C) 2010 American Institute of Physics. [doi:10.1063/1.3507256]
\end{abstract}

\section{INTRODUCTION}

Because of recent progress in generation techniques of intense terahertz $(\mathrm{THz})$ radiation pulses,${ }^{1-3}$ we are now able to observe various nonlinear phenomena in $\mathrm{THz}$ spectral region using those intense $\mathrm{THz}$ pulses. Time-domain measurements, especially, are expected to supply rich spectroscopic information of the system as conventional linear time-domain $\mathrm{THz}$ spectroscopies. Nonlinear transmission of intense $\mathrm{THz}$ waves has been observed in electronic systems in different types of semiconductors. ${ }^{4-9}$ More recently, nonlinearity in vibrational $^{10}$ and phonon ${ }^{11}$ systems has been observed.

Using nonlinear spectroscopy, ${ }^{12,13}$ various types of information that is not accessed by linear spectroscopic measurements can be obtained. Accessible information includes dynamics of population and coherence, inhomogeneous distribution of resonance frequency, anharmonicity of intra- and intermolecular vibrational modes, coupling between different modes, and interaction between neighboring molecules. In the optical frequency region, variety of techniques have been developed and applied for this purpose. Even when limited to time-resolved or time-domain techniques, a wealth of information has been obtained using spectroscopic measurements such as pump-probe method, photon echoes, transient grating, optical Kerr effect, transient hole burning, coherent Raman scattering, impulsive Raman scattering, impulsive phonon excitation, other various types of four-wave mixing, two-photon absorption, and double resonance spectroscopy.

In the $\mathrm{THz}$ frequency region, however, it is not established yet what types of spectroscopic measurements can be performed and what kind of information is obtained from them in the nonlinear regime. In experiments using a single

a)Electronic mail: hattori@bk.tsukuba.ac.jp. intense $\mathrm{THz}$ pulse, extraction of nonlinear signal is not easy, and only limited information is obtained from the experimental signals. By adding another $\mathrm{THz}$ pulse, extraction of nonlinear signals becomes easier, and rich spectroscopic information can be obtained from the dependence of the signal on the time interval between the two pulses. In this report, two-pulse measurements are studied as a model experiment, where two $\mathrm{THz}$ pulses are incident on the sample medium, and the waveform of the transmitted $\mathrm{THz}$ pulse is measured.

It is assumed, in the theoretical consideration, that only nonlinear contribution to the transmitted $\mathrm{THz}$ pulse waveform affected by both of the two incident $\mathrm{THz}$ pulses is extracted in the measurements by using appropriate experimental techniques. This assumption enables a perturbative approach in the theoretical analysis. Since the signal is a function of the pulse interval and the observation time, the measurement is inherently a two-dimensional (2D) one. 2D spectroscopy in frequency and time domain has been applied for studies of vibrational and other excitation modes, ${ }^{14,15}$ and proved to be powerful in elucidating dynamics, inhomogeneity, and nonlinear coupling between modes. 2D $\mathrm{THz}$ spectroscopy was first proposed by Okumura and Tanimura, and theoretically shown that it can give information on anharmonicity of lowfrequency modes in liquid water ${ }^{16}$ and a free energy landscape of a dipolar crystal system. ${ }^{17}$

Second-order and third-order nonlinear $\mathrm{THz}$ spectroscopies with two incident $\mathrm{THz}$ pulses will be discussed in this study. When the system does not have inversion symmetry, second-order nonlinearity is the most important, whereas third order is the lowest when there is inversion symmetry. The second-order 2D THz spectroscopy has close correspondence with fifth-order $2 \mathrm{D}$ Raman spectroscopy, ${ }^{18}$ as described in more detail in Sec. II C. Since the proposal by Tanimura and Mukamel, ${ }^{19}$ extensive studies on fifth-order 
2D Raman spectroscopy have been reported on theories, ${ }^{19-25}$ experiments, ${ }^{26-37}$ and calculations. ${ }^{38-45}$ These studies have clarified that information on inhomogeneity, nonlinearity origin, mode coupling, and intermolecular interaction can be obtained from the 2D spectroscopy. It has also been shown that multidimensional nonlinear response of classical systems has a wealth of information on chaotic properties of the system. ${ }^{46}$ Second-order 2D THz spectroscopy should share exactly the same ability as fifth-order 2D Raman spectroscopy because of the theoretical correspondence between them. Third-order 2D THz spectroscopy, ${ }^{16,17}$ on the other hand, is related to but does not have exact correspondence with seventh-order Raman spectroscopy, since seventh-order Raman signals inevitably contains contributions from lower-order nonlinearities, whereas third-order $\mathrm{THz}$ spectroscopy does not in centrosymmetric media.

2D spectroscopy in the midinfrared (MIR) (Refs. 14 and 15) region is also a powerful tool for the study of dynamics and nonlinearity of vibrational modes. MIR 2D spectroscopy, however, is different from that in the $\mathrm{THz}$ region in two points. First, the vibrational mode in MIR has essentially a quantum mechanical nature while they are much more classical in $\mathrm{THz}$ region. Second, time resolution of measurements is lower than the vibrational period in MIR whereas time-resolved acquisition of oscillation waveforms is the particular feature of the $\mathrm{THz}$ time-domain measurements.

The theoretical treatment in the present study is purely classical and phenomenological. ${ }^{23,24}$ Sources of weak nonlinearities are introduced into a classical oscillator described by the Lorentz model. In the first paper on 2D Raman spectroscopy ${ }^{19}$ and in succeeding papers by Tanimura's group, ${ }^{16,20-22}$ a quantum mechanical treatment was used for the derivation of the expression of nonlinear responses, and tedious procedures were taken for the derivation of the analytical expression for the signal response. Their studies, however, are focused on systems with weak anharmonicity and/or weak nonlinear coupling. Their model, therefore, of the material system is almost of a classical nature, and it will be shown that the theoretical expressions obtained in their study agree exactly with those of the present one. Since in $\mathrm{THz}$ region the photon energy is much smaller than the temperature, i.e., $\hbar \omega \ll k T$, the systems have a classical nature in most cases. By using a classical theory, therefore, the description and analysis of the experiments is closed in classical physics. The pure classical presentation in the present study is related to the study of Jansen and Mukamel, ${ }^{47}$ where it was shown how multiple-time response functions can be factorized into products of single-time response functions. Furthermore, secondorder nonlinear $\mathrm{THz}$ responses, which corresponds to fifthorder Raman responses, consist of only coherent processes, and do not contain contributions of diagonal elements of the density matrix of the system. In this case, a classical model is sufficient for the complete description of system responses. This is equivalent to the fact that the linear optical response of a harmonic oscillator can be described completely using a classical Lorentz model.

The aim of this study is to elucidate what kind of information can be obtained directly from experimental results without depending on the model of the system. The classical and phenomenological description of the system, adopted in the present study, is the simplest and best for this purpose. The major target of the present study is vibrational and phonon systems since rich information can be obtained using 2D spectroscopy from systems with narrow spectral widths, although the present theoretical treatment can also be applied to electronic and other types of systems if the nonlinearity of the system shares the same nature.

The outline of this paper is described briefly. In Sec. II, first I describe experiments under consideration and experimental requirement for the observation of relevant nonlinear signals. Then framework of the theoretical treatment is outlined. Finally, fifth-order and seventh-order Raman 2D spectroscopies are reviewed in view of the correspondence with and difference from second-order and third-order $\mathrm{THz} 2 \mathrm{D}$ spectroscopy. Derivation of various expressions and numerical calculation results are presented for the second-order processes in Sec. III, and for the third-order processes in Sec. IV. Discussion on the results obtained and also on possible extension of the present theoretical treatment is given in Sec. V. Finally, the paper is concluded in Sec. VI.

\section{MODEL}

\section{A. Model experiment}

The experiment under consideration is described here. Two intense THz pulses, $E_{1}$ and $E_{2}$, are incident on a nonlinear medium at times $-T_{1}$ and 0 . THz electric field generated from nonlinear polarization induced by both of $E_{1}$ and $E_{2}$ is observed at time $t$. The time sequence of the incident pulses and the probe is schematically depicted in Fig. 1. The signal field is obtained as a function of $T_{1}$ and $t$, which composes the two-dimensional time-domain signal. It is expected that information that is not accessed by linear spectroscopy is obtained from the nonlinear spectroscopy. When the nonlinear media are noncentrosymmetric, the media have second-order nonlinear optical effects. In this case, the second-order signal is observed. When the system is centrosymmetric, the lowestorder nonlinearity is the third-order one. We observe, in this case, the electric field of the third order.

In either case of second-order or third-order nonlinearity, it is assumed in the following analysis that only the nonlinear field influenced by both $E_{1}$ and $E_{2}$ is observed. The electric field at time $t$ contains contributions by linearly propagated field of $E_{1}$ and $E_{2}$, nonlinear polarization generated only by either $E_{1}$ or $E_{2}$, and that generated by both of $E_{1}$ and $E_{2}$. In nonlinear optical spectroscopy in the visible region, noncollinear configuration of the two pump beams is

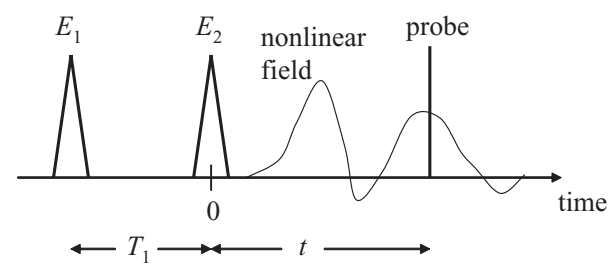

FIG. 1. Timing of the incident $\mathrm{THz}$ pulses, $E_{1}$ and $E_{2}$, and the probe in the two-dimensional time-domain $\mathrm{THz}$ spectroscopy. 
usually adopted for the picking up of the last contribution. In $\mathrm{THz}$ region, however, collinear geometry is usually preferred because the $f$ numbers of the pump waves are small. Techniques, therefore, other than the noncollinear configuration should be introduced for the separation. Photon echo signal in the visible region has been observed in the collinear configuration using a two-frequency modulation method, ${ }^{48-50}$ where two pump beams are modulated at different frequencies and sum-frequency component in the transmitted light intensity is observed. This or similar technique will enable detection of only the nonlinear field contributed by both $E_{1}$ and $E_{2}$.

\section{B. Theoretical model}

The linear equation of motion of a vibrational mode coupled to the incident $\mathrm{THz}$ radiation is expressed in the Lorentz model as

$$
m\left[\frac{d^{2}}{d t^{2}} x(t)+2 \Gamma \frac{d}{d t} x(t)+\Omega^{2} x(t)\right]=q_{0} E(t) .
$$

Here, $E(t)$ is the electric field of the incident $\mathrm{THz}$ radiation, and $x(t), m, \Gamma, \Omega$, and $q_{0}$ are the coordinate, mass, damping constant, resonance frequency, and effective charge of the vibrational mode. Polarization $P(t)$ is expressed as

$$
P(t)=N p(t)=N q_{0} x(t),
$$

where $N$ is the density of the molecule, and $p$ is the effective electric dipole moment of the mode. By solving Eq. (1), the linear polarization can be expressed in terms of the linear response function of polarization, $r(t)$, as

$$
\begin{aligned}
& P(t)=\int_{0}^{\infty} d t^{\prime} r\left(t^{\prime}\right) E\left(t-t^{\prime}\right), \\
& r(t)=\frac{N q_{0}^{2}}{m} \cdot \frac{1}{\zeta} e^{-\Gamma t} \sin \zeta t \quad(t>0),
\end{aligned}
$$

where

$$
\zeta=\left(\omega_{0}^{2}-\Gamma^{2}\right)^{1 / 2}
$$

The response function $r(t)$ is sometimes referred to the Green's function or the propagator. ${ }^{24}$ Underdamped oscillation condition $\left(\Gamma<\omega_{0}\right)$ is assumed here although this model can also be applied to critically damped or overdamped oscillators.

The incident electric field $E(t)$ is assumed to be a sum of the first and second incident $\mathrm{THz}$ field:

$$
E(t)=E_{1}(t)+E_{2}(t)
$$

and

$$
E_{1}(t)=E_{2}\left(t+T_{1}\right)
$$

where $E_{2}(t)$ is peaked at $t=0$, and later in the numerical evaluation, approximated by a delta function. Here, $E_{1}$ and $E_{2}$ are assumed to have the same sign and amplitude. A case with different signs is also considered in the discussion of third-order nonlinearity since the third-order nonlinear field is affected by the relative sign and amplitude of $E_{1}$ and $E_{2}$.
When there is nonlinearity of any type in the system, nonlinear electric field $E^{\mathrm{NL}}$ is radiated from the nonlinear polarization $P^{\mathrm{NL}}$ according to the one-dimensional propagation equation:

$$
\frac{\partial^{2}}{\partial z^{2}} E(z, t)=\epsilon \mu_{0} \frac{\partial^{2}}{\partial t^{2}} E(z, t)+\mu_{0} \frac{\partial^{2}}{\partial t^{2}} P^{\mathrm{NL}}(z, t) .
$$

By assuming slowly-varying envelope approximation, we obtain

$$
E^{\mathrm{NL}}(t)=-\frac{Z_{0} L}{2 n} \frac{d}{d t} P^{\mathrm{NL}}(t)
$$

Here, $L$ is the interaction length, $Z_{0} \equiv\left(\mu_{0} / \epsilon_{0}\right)^{1 / 2}=377 \Omega$ the vacuum impedance, and $n$ the refractive index of the medium. In the derivation of this expression, it is assumed that the refractive index does not depend on frequency, and the deformation of field waveform due to linear absorption is neglected.

We use the perturbation method, where $x$ and $P$ are expanded in the order of $E$ as

$$
\begin{aligned}
& x=x^{(1)}+x^{(2)}+x^{(3)}, \\
& P=P^{(1)}+P^{(2)}+P^{(3)},
\end{aligned}
$$

and

$$
x^{(n)}, P^{(n)} \propto E^{n} \quad(n=1,2,3) .
$$

In noncentrosymmetric media, only the second-order terms are retained since the higher-order terms are smaller in the perturbation regime. In centrosymmetric media, on the other hand, the second-order terms vanish.

Corresponding to three parameters, $\Omega, q_{0}$, and $\Gamma$ in Eq. (1), three types of nonlinearities are introduced into the equations above. These three are anharmonicity (AH), nonlinear coupling (NC), and nonlinear damping (ND). Details of them are described in Secs. III and IV.

For numerical evaluation, a single-mode system with $\Gamma / \Omega=0.1$ is considered throughout this paper, and the duration of the incident $\mathrm{THz}$ pulses is assumed to be much shorter than the vibrational period.

\section{Fifth-order and seventh-order Raman scattering}

Fifth-order and seventh-order Raman scattering processes are briefly described here since second-order and third-order two-dimensional $\mathrm{THz}$ spectroscopy have close correspondence with the fifth-order and seventh-order twodimensional Raman spectroscopy. ${ }^{16}$

The linear equation of motion and the polarization for a coordinate of an electronically off-resonance Raman active vibrational mode are expressed as

$$
m\left[\frac{d^{2}}{d t^{2}} x(t)+2 \Gamma \frac{d}{d t} x(t)+\Omega^{2} x(t)\right]=\left(\frac{\partial}{\partial x} \alpha\right)[E(t)]^{2}
$$

and

$$
P(t)=N \alpha E(t)
$$


Here, $\alpha$ is the molecular polarizability. We can see the exact correspondence with the $\mathrm{THz}$ response by replacing $\alpha$ and $[E(t)]^{2}$ with $p$ and $E(t)$.

The experimental signal in the fifth-order Raman spectroscopy is expressed in terms of the three-time correlation function

$$
R_{\alpha}^{(3)}=\left\langle\left[[\alpha(t), \alpha(0)], \alpha\left(-T_{1}\right)\right]\right\rangle,
$$

in the quantum mechanical description, where $\alpha(t)$ is the polarizability operator. This correlation function contains information on the polarizability response function. In the second-order nonlinear $\mathrm{THz}$ spectroscopy, on the other hand, the observed signal is expressed in terms of the three-time correlation function

$$
R_{\mu}^{(3)}=\left\langle\left[[\mu(t), \mu(0)], \mu\left(-T_{1}\right)\right]\right\rangle,
$$

where $\mu(t)$ is the electric dipole moment operator. The dynamics of polarization and polarizability are not necessarily the same for a specific material system, but the equivalence of the theoretical treatments of these two type of nonlinear spectroscopies implies that a similar type of information is obtained from them.

There are, however, some differences between these two types of spectroscopies. When the excitation and probe optical pulses are sufficiently short, the signal field observed in the nonlinear $\mathrm{THz}$ spectroscopy is proportional to the time derivative of the nonlinear polarization, which is expressed by Eq. (16), as described by Eq. (9). In the fifth-order Raman spectroscopy, on the other hand, observed signals are proportional to the nonlinear polarizability itself when optical heterodyne detection is used, ${ }^{31,34,35}$ which is expressed by the polarizability correlation function [Eq. (15)]. In homodyne detection measurements, the observed signals are proportional to the squared magnitude of the polarizability.

A difficulty in fifth-order nonlinear spectroscopy is that the signals can be very easily contaminated by cascades of third-order processes. Blank et al. pointed out that all the earlier experimental results ${ }^{26-28}$ on fifth-order Raman spectroscopy are dominated by cascaded thirdorder processes and no information on the intrinsic fifthorder response is included. ${ }^{30}$ Special techniques, such as optical heterodyning, ${ }^{31,34,35}$ a special phase matching configuration, ${ }^{32,33}$ or a special polarization configuration, ${ }^{51,52}$ were introduced to obtain purely fifth-order signals. The second-order $\mathrm{THz}$ spectroscopy does not have this difficulty since it is the lowest-order nonlinear process.

Although seventh-order Raman spectroscopy and thirdorder $\mathrm{THz}$ spectroscopy have a relationship similar to that of fifth-order Raman and second-order $\mathrm{THz}$ spectroscopy, seventh-order Raman scattering signals suffer worse from influences of lower-order nonlinearities. Cho et al. pointed out that it is hard to experimentally measure the pure seventhorder Raman scattering signal without contamination from cascaded contributions of lower-order responses. ${ }^{53}$ Furthermore, seventh-order responses, i.e., seventh-order susceptibilities, themselves have contributions of lower order nonlinearities in the system Hamiltonian. This is because interaction of any order is allowed for Raman-active modes. In contrast, for third-order $\mathrm{THz}$ spectroscopy, especially in centrosymmetric media, the signal does not suffer from influences of cascaded lower-order nonlinear processes or internal lower-order nonlinearities.

\section{SECOND-ORDER PROCESSES}

When parameters in the equation of motion of the system, Eq. (1), and the polarization, Eq. (2), are all constants, the response of the system to the incident field is linear, and there are no nonlinear phenomena. Nonlinearity of the system can arise from $x$ dependence of the resonance frequency, $\Omega$, the charge, $q_{0}$, or the damping constant, $\Gamma$. These three cases correspond to three sources of nonlinearity, i.e., anharmonicity $(\mathrm{AH})$, nonlinear coupling (NC), and nonlinear damping (ND), respectively. Each case is considered below.

In the first paper on fifth-order Raman spectroscopy, ${ }^{19}$ only NC was considered as a source of nonlinearity. Later it was well understood that $\mathrm{AH}$ and $\mathrm{NC}$ can be the source of nonlinearity. ${ }^{22}$ ND has not been studied before. Since the damping constant can be regarded as the imaginary part of energy, it can be regarded as a generalization of the $\mathrm{AH}$ case. In general, the three mechanisms coexist in the system, and the nonlinear response is a sum of their contributions. Simulation studies on fifth-order Raman response of liquid $\mathrm{CS}_{2}$ and $\mathrm{CS}_{2}$ dissolved in Xe showed that $\mathrm{AH}$ and $\mathrm{NC}$ mechanisms contribute differently to the nonlinear response. ${ }^{42,54}$

\section{A. Anharmonicity}

The lowest order anharmonicity is introduced by adding a third-order term to the harmonic potential function of the vibrational mode as

$$
V(x)=m\left(\frac{1}{2} \Omega^{2} x^{2}+\frac{D_{3}}{3} x^{3}\right) .
$$

Then the last term in the left-hand side of Eq. (1) changes as

$$
m \omega_{0}^{2} x \rightarrow \frac{\partial}{\partial x} V(x)=m\left(\Omega^{2} x+D_{3} x^{2}\right) .
$$

The procedure of the derivation of expressions in the perturbation method is briefly described below. First the vibrational coordinate, $x$, is expanded in power series of $E(t)$, as in Eq. (10), up to the second order, and substituted into the equation of motion, Eq. (1), with a nonlinear term in the potential expressed by Eq. (18). Then by picking up terms proportional to the first or the second order of $E(t)$, we obtain equations for $x^{(1)}(t)$

$$
\frac{d^{2}}{d t^{2}} x^{(1)}(t)+2 \Gamma \frac{d}{d t} x^{(1)}(t)+\Omega^{2} x^{(1)}(t)=\frac{q_{0}}{m} E(t),
$$

and for $x^{(2)}(t)$

$$
\frac{d^{2}}{d t^{2}} x^{(2)}(t)+2 \Gamma \frac{d}{d t} x^{(2)}(t)+\Omega^{2} x^{(2)}(t)=-D_{3}\left\{x^{(1)}(t)\right\}^{2} .
$$

From them, solutions for the first- and second-order polarization are obtained as

$$
P^{(1)}(t)=\int_{0}^{\infty} d t^{\prime} r\left(t^{\prime}\right) E\left(t-t^{\prime}\right),
$$




$$
P^{(2)}(t)=-\frac{m D_{3}}{N^{2} q_{0}^{3}} \int_{0}^{\infty} d t^{\prime} r\left(t^{\prime}\right)\left\{P^{(1)}\left(t-t^{\prime}\right)\right\}^{2}
$$

Corresponding to the selective detection of the contribution of both $E_{1}$ and $E_{2}$ to the nonlinear field in the experiments, only cross terms of $E_{1}$ and $E_{2}$ are picked up from the righ-hand side of Eq. (22). Then we obtain the expression for the second-order polarization as

$$
\begin{aligned}
P^{(2)}(t)= & -\frac{2 m D_{3}}{N^{2} q_{0}^{3}} \int_{0}^{\infty} d t^{\prime} \int_{0}^{\infty} d t_{1} \int_{0}^{\infty} d t_{2} \\
& \times r\left(t^{\prime}\right) r\left(t_{1}\right) r\left(t_{2}\right) E_{2}\left(t-t^{\prime}-t_{1}\right) E_{2}\left(t-t^{\prime}-t_{2}+T_{1}\right) .
\end{aligned}
$$
function

When the incident electric field can be regarded as a delta

$$
E_{2}(t)=E_{0} \delta(t)
$$

Equation (23) is reduced to

$$
P_{\mathrm{AH}}^{(2)}\left(T_{1}, t\right)=-\frac{2 m D_{3}}{N^{2} q_{0}^{3}} E_{0}^{2} \int_{0}^{t} d t^{\prime} r\left(t^{\prime}\right) r\left(t-t^{\prime}\right) r\left(t+T_{1}-t^{\prime}\right)
$$

Because of the exact theoretical correspondence between the second-order $\mathrm{THz}$ response and the fifth-order Raman response, the fifth-order Raman response function, $R_{\mathrm{AH}}^{(5)}\left(T_{1}, t\right)$, is expressed, under the current treatment, as

$R_{\mathrm{AH}}^{(5)}\left(T_{1}, t\right) \propto-\int_{0}^{t} d t^{\prime} r_{R}\left(t^{\prime}\right) r_{R}\left(t-t^{\prime}\right) r_{R}\left(t+T_{1}-t^{\prime}\right)$.

Here $r_{R}(t)$ is the Raman response function of the relevant mode. This expression agrees with Eq. (4.9) of Ref. 22, which describes the fifth-order Raman response obtained using the quantum mechanical theory. Its analytical expression has also been given. ${ }^{22}$ According to Eq. (9), the second-order field is emitted from the second-order polarization as

$$
\begin{aligned}
E_{\mathrm{AH}}^{(2)}\left(T_{1}, t\right) & =-\frac{Z_{0} L}{2 n} \frac{d}{d t} P_{\mathrm{AH}}^{(2)}\left(T_{1}, t\right) \\
& \propto \frac{d}{d t} \int_{0}^{t} d t^{\prime} r\left(t^{\prime}\right) r\left(t-t^{\prime}\right) r\left(t+T_{1}-t^{\prime}\right) .
\end{aligned}
$$

Numerical calculations for $P_{\mathrm{AH}}^{(2)}\left(T_{1}, t\right)$, which is equivalent to $R_{\mathrm{AH}}^{(5)}\left(T_{1}, t\right)$, and $E_{\mathrm{AH}}^{(2)}\left(T_{1}, t\right)$ are shown in Figs. 2(a) and 2(b). The damping constant is set at $\Gamma / \Omega=0.1$ for the calculations throughout this paper.

\section{B. Nonlinear coupling}

Another source of nonlinearity is a nonlinear dependence of the polarization, $p$, on the coordinate. This type of nonlinearity is referred to as "nonlinear coupling" since this is the nonlinearity in the coupling between the vibrational mode and the radiation field. In the fifth-order Raman response, nonlinear coupling is caused by coordinate dependence of transition dipole moment (non-Condon effect), which is important for (a)

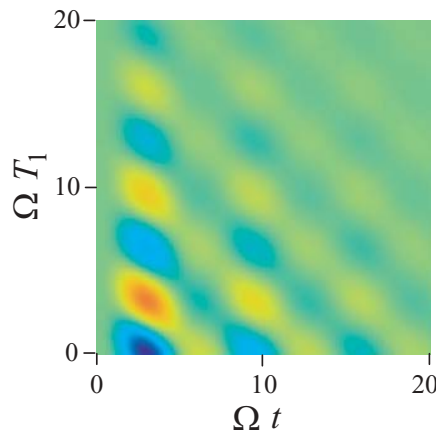

(c)

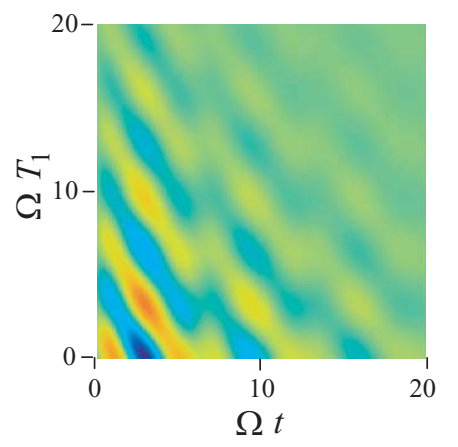

FIG. 2. Two-dimensional profiles of second-order signals for a system with an anharmonic potential and nonlinear damping. (a) Second-order polarization for an anharmonic potential, which corresponds to the response function for the fifth-order Raman scattering with an anharmonic potential. (b) Second-order $\mathrm{THz}$ field for an anharmonic potential. (c) Second-order $\mathrm{THz}$ field for nonlinear damping. Each image is normalized to its maximum magnitude.

the description of ultrafast spectroscopic measurements. ${ }^{55,56}$ A higher-order term is introduced to the dipole moment as

$$
p=q_{0} x+\frac{1}{2} q_{1} x^{2}
$$

Then the right-hand side of Eq. (1) becomes as

$$
q_{0} E \rightarrow\left(\frac{\partial}{\partial x} p\right) E=\left(q_{0}+q_{1} x\right) E,
$$

and the polarization expressed by Eq. (2) also comes to contain a second-order term accordingly.

By using the perturbational method as described above, we obtain

$$
\begin{aligned}
& x^{(1)}(t)=\frac{1}{N q_{0}} \int_{0}^{\infty} d t^{\prime} r\left(t^{\prime}\right) E\left(t-t^{\prime}\right), \\
& x^{(2)}(t)=\frac{q_{1}}{N q_{0}^{2}} \int_{0}^{\infty} d t^{\prime} r\left(t^{\prime}\right) x^{(1)}\left(t-t^{\prime}\right) E\left(t-t^{\prime}\right),
\end{aligned}
$$

and

$$
\begin{aligned}
P^{(2)}(t)= & N q_{0} x^{(2)}(t)+\frac{1}{2} N q_{1}\left[x^{(1)}(t)\right]^{2} \\
= & \frac{q_{1}}{N q_{0}^{2}} \int_{0}^{\infty} d t^{\prime} \int_{0}^{\infty} d t^{\prime \prime} r\left(t^{\prime}\right) r\left(t^{\prime \prime}\right) \\
& \times E\left(t-t^{\prime}\right)\left[E\left(t-t^{\prime}-t^{\prime \prime}\right)+\frac{1}{2} E\left(t-t^{\prime \prime}\right)\right] .
\end{aligned}
$$


The second-order polarization consists of two contributions. The first and second terms in Eq. (32) correspond to the effect of nonlinearity in the excitation process of the vibrational mode and that in the emission process of $\mathrm{THz}$ waves, respectively. obtain

When picking up only the cross term of $E_{1}$ and $E_{2}$, we

$$
\begin{aligned}
P^{(2)}(t)= & \frac{q_{1}}{N q_{0}^{2}} \int_{0}^{\infty} d t^{\prime} \int_{0}^{\infty} d t^{\prime \prime} r\left(t^{\prime}\right) r\left(t^{\prime \prime}\right) \\
& \times\left[E_{2}\left(t-t^{\prime}\right) E_{2}\left(t+T_{1}-t^{\prime}-t^{\prime \prime}\right)\right. \\
& +E_{2}\left(t+T_{1}-t^{\prime}\right) E_{2}\left(t-t^{\prime}-t^{\prime \prime}\right) \\
& \left.+E_{2}\left(t-t^{\prime}\right) E_{2}\left(t+T_{1}-t^{\prime \prime}\right)\right]
\end{aligned}
$$

When the incident electric field is regarded as a delta function, as expressed in Eq. (24), we obtain

$$
P_{\mathrm{NC}}^{(2)}\left(T_{1}, t\right)=\frac{q_{1}}{N q_{0}^{2}} E_{0}^{2} r(t)\left[r\left(T_{1}\right)+r\left(t+T_{1}\right)\right] .
$$

This corresponds to the fifth-order Raman response function for nonlinear coupling

$$
R_{\mathrm{NC}}^{(5)}\left(T_{1}, t\right) \propto r_{R}(t)\left[r_{R}\left(T_{1}\right)+r_{R}\left(t+T_{1}\right)\right] .
$$

This expression agrees with that obtained based on a quantum mechanical treatment, which appears in Eq. (4.10) of Ref. 22 and in the first term of Eq. (3.16) of Ref. 19. The second-order $\mathrm{THz}$ field emitted is expressed as

$$
E_{\mathrm{NC}}^{(2)}\left(T_{1}, t\right) \propto-\frac{d}{d t}\left\{r(t)\left[r\left(T_{1}\right)+r\left(t+T_{1}\right)\right]\right\} .
$$

Numerical calculations for $P_{\mathrm{NC}}^{(2)}\left(T_{1}, t\right)$ and $E_{\mathrm{NC}}^{(2)}\left(T_{1}, t\right)$ are shown in Fig. 3.

\section{Nonlinear damping}

A third source of nonlinearity is a dependence of the damping constant on the coordinate, as expressed by

$$
\Gamma \rightarrow \Gamma+\Gamma_{1} x .
$$

(a)

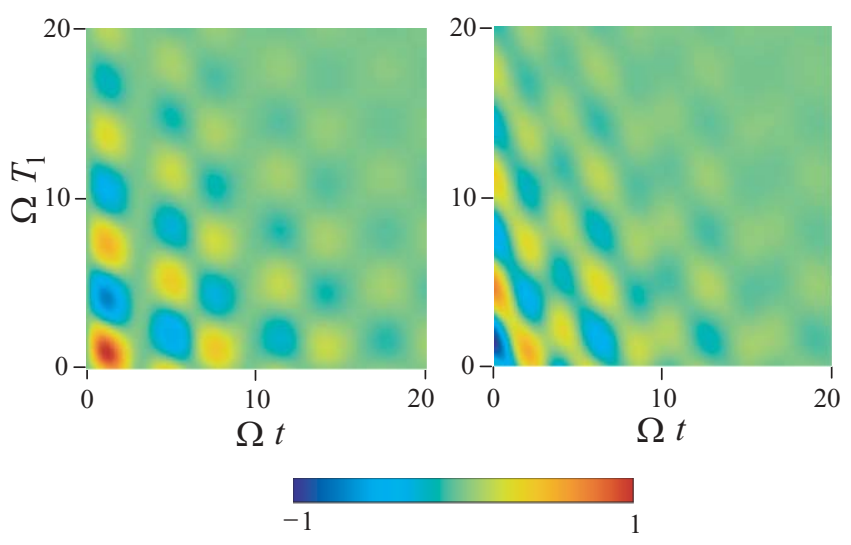

FIG. 3. Second-order two-dimensional signals for systems with nonlinear coupling. (a) Second-order polarization for nonlinear coupling, which corresponds to the response function for the fifth-order Raman scattering with nonlinear coupling. (b) Second-order $\mathrm{THz}$ field for the nonlinear coupling case. Each image is normalized to its maximum magnitude.
Using the perturbation method, we obtain

$$
P^{(1)}(t)=\int_{0}^{\infty} d t^{\prime} r\left(t^{\prime}\right) E\left(t-t^{\prime}\right)
$$

and

$$
P^{(2)}(t)=-\frac{m \Gamma_{1}}{N^{2} q_{0}^{3}} \frac{d}{d t} \int_{0}^{\infty} d t^{\prime} r\left(t^{\prime}\right)\left\{P^{(1)}\left(t-t^{\prime}\right)\right\}^{2} .
$$

This expression shows that the second-order polarization of ND case is proportional to the time derivative of the secondorder polarization of the $\mathrm{AH}$ case. By picking up only the cross term of $E_{1}$ and $E_{2}$, we obtain

$$
\begin{aligned}
P^{(2)}(t)= & -\frac{2 m \Gamma_{1}}{N^{2} q_{0}^{3}} \frac{d}{d t} \int_{0}^{\infty} d t^{\prime} \int_{0}^{\infty} d t_{1} \int_{0}^{\infty} d t_{2} \\
& \times r\left(t^{\prime}\right) r\left(t_{1}\right) r\left(t_{2}\right) E_{2}\left(t-t^{\prime}-t_{1}\right) E_{2}\left(t-t^{\prime}-t_{2}+T_{1}\right) .
\end{aligned}
$$

When delta function incident $\mathrm{THz}$ pulses are assumed, this equation becomes

$$
\begin{aligned}
P_{\mathrm{ND}}^{(2)}\left(T_{1}, t\right)= & -\frac{2 m \Gamma_{1}}{N^{2} q_{0}^{3}} E_{0}^{2} \frac{d}{d t} \\
& \times \int_{0}^{t} d t^{\prime} r\left(t^{\prime}\right) r\left(t-t^{\prime}\right) r\left(t+T_{1}-t^{\prime}\right) .
\end{aligned}
$$

This is proportional to $E_{\mathrm{AH}}^{(2)}\left(T_{1}, t\right)$. The emitted nonlinear field is expressed as

$$
E_{\mathrm{ND}}^{(2)}\left(T_{1}, t\right) \propto \frac{d^{2}}{d t^{2}} \int_{0}^{t} d t^{\prime} r\left(t^{\prime}\right) r\left(t-t^{\prime}\right) r\left(t+T_{1}-t^{\prime}\right) .
$$

A numerical result for $E_{\mathrm{ND}}^{(2)}\left(T_{1}, t\right)$ is shown in Fig. 2(c).

\section{THIRD-ORDER PROCESSES}

In this section, two-dimensional signal profiles of thirdorder nonlinear spectroscopy in centrosymmetric media are described. Third-order nonlinear field is proportional to the third power of incident $\mathrm{THz}$ field. Three incident pulses can be used in general for third-order time-resolved measurements. Then the signals are observed as a function of three temporal intervals among the incident $\mathrm{THz}$ pulses and the probe, leading to three-dimensional spectroscopy. In this study, however, we limit discussions to measurements with two incident $\mathrm{THz}$ pulses for simplicity. With two incident pulses, the third-order signals consist of two contributions in collinear geometry. One is proportional to the first incident field and to the square of the second incident field. This corresponds to a sequence of three pulses at times $-T_{1}, 0$, and 0 , which is referred to as $\left(T_{1}, 0, t\right)$ sequence in the following by using time intervals between pulses. The other contribution is that proportional to the second incident field and to the square of the first incident field, which is referred to as $\left(0, T_{1}, t\right)$ sequence. These pulse timings are depicted in Fig. 4. Separation of these two contributions in experiments is, in principle, possible since the dependence of the signal on the two incident fields are different. When, for example, the sign of the field of the second incident pulse is inverted, the sign of the $\left(0, T_{1}, t\right)$ 
(a)

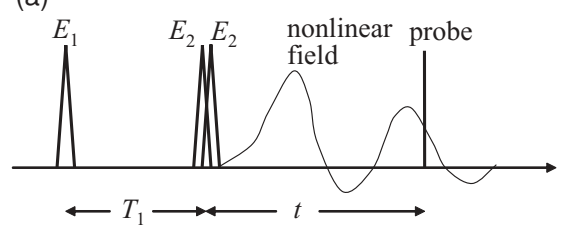

(b)

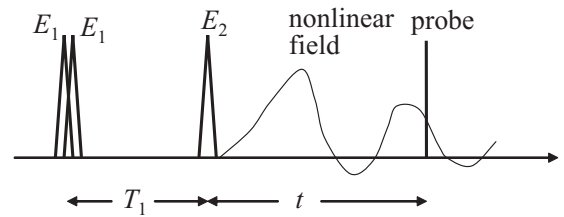

FIG. 4. Two contributions for third-order two-dimensional signals in collinear geometry. (a) $\left(T_{1}, 0, t\right)$ sequence. (b) $\left(0, T_{1}, t\right)$ sequence.

contribution is inverted, but that of the $\left(T_{1}, 0, t\right)$ contribution does not.

In the following three sources of third-order nonlinearity, AH, NC, and that of two-level systems, are considered. In either case, the nonlinearity order that is introduced in the second-order nonlinearity case is not allowed because of the inversion symmetry of the system. This is markedly different from the case of seventh-order Raman spectroscopy. In third-order $\mathrm{THz}$ spectroscopy, the signals exhibit only the features of the lowest-order allowed nonlinearity, whereas in the seventh-order Raman spectroscopy, the signals are inevitably contaminated by influences of lower-order nonlinearities of the system.

\section{A. Anharmonicity}

In centrosymmetric media, the lowest-order anharmonicity is expressed by a fourth-order term in the potential of the vibrational mode as

$$
V(x)=m\left(\frac{1}{2} \Omega^{2} x^{2}+\frac{D_{4}}{4} x^{4}\right) .
$$

Then the last term in the left-hand side of Eq. (1) changes as

$$
m \omega_{0}^{2} x \rightarrow \frac{\partial}{\partial x} V(x)=m\left(\Omega^{2} x+D_{4} x^{3}\right) .
$$

The equation for $x^{(1)}(t)$ is the same as Eq. (19). For $x^{(3)}(t)$ we obtain

$$
\frac{d^{2}}{d t^{2}} x^{(3)}(t)+2 \Gamma \frac{d}{d t} x^{(3)}(t)+\Omega^{2} x^{(3)}(t)=-D_{4}\left\{x^{(1)}(t)\right\}^{3} .
$$

Solutions for the first- and third-order polarization are obtained as

$$
\begin{aligned}
& P^{(1)}(t)=\int_{0}^{\infty} d t^{\prime} r\left(t^{\prime}\right) E\left(t-t^{\prime}\right) \\
& P^{(3)}(t)=-\frac{m D_{4}}{N^{3} q_{0}^{4}} \int_{0}^{\infty} d t^{\prime} r\left(t^{\prime}\right)\left\{P^{(1)}\left(t-t^{\prime}\right)\right\}^{3} .
\end{aligned}
$$

By assuming delta-function incident $\mathrm{THz}$ pulses, the contributions of $\left(T_{1}, 0, t\right)$ and $\left(0, T_{1}, t\right)$ sequences to the thirdorder polarization are obtained as

$$
\begin{aligned}
P_{\mathrm{AH}}^{(3)}\left(T_{1}, 0, t\right)= & -\frac{3 m D_{4}}{N^{3} q_{0}^{4}} E_{0}^{3} \\
& \times \int_{0}^{t} d t^{\prime} r\left(t^{\prime}\right)\left[r\left(t-t^{\prime}\right)\right]^{2} r\left(t+T_{1}-t^{\prime}\right)
\end{aligned}
$$

and

$$
\begin{aligned}
P_{\mathrm{AH}}^{(3)}\left(0, T_{1}, t\right)= & -\frac{3 m D_{4}}{N^{3} q_{0}^{4}} E_{0}^{3} \\
& \times \int_{0}^{t} d t^{\prime} r\left(t^{\prime}\right) r\left(t-t^{\prime}\right)\left[r\left(t+T_{1}-t^{\prime}\right)\right]^{2},
\end{aligned}
$$

respectively.

Contributions of the $\left(T_{1}, 0, t\right)$ and $\left(0, T_{1}, t\right)$ sequences to the third-order $\mathrm{THz}$ field are expressed as

$E_{\mathrm{AH}}^{(3)}\left(T_{1}, 0, t\right) \propto \frac{d}{d t} \int_{0}^{t} d t^{\prime} r\left(t^{\prime}\right)\left[r\left(t-t^{\prime}\right)\right]^{2} r\left(t+T_{1}-t^{\prime}\right)$

and

$$
E_{\mathrm{AH}}^{(3)}\left(0, T_{1}, t\right) \propto \frac{d}{d t} \int_{0}^{t} d t^{\prime} r\left(t^{\prime}\right) r\left(t-t^{\prime}\right)\left[r\left(t+T_{1}-t^{\prime}\right)\right]^{2} .
$$

Numerical calculation results for the third-order $\mathrm{THz}$ field are shown in Fig. 5. Panels (a) and (b) show the contributions of $\left(T_{1}, 0, t\right)$ and $\left(0, T_{1}, t\right)$ sequences, respectively. Panel (c) shows the sum of (a) and (b), which is achieved when the two incident $\mathrm{THz}$ pulses have the same sign and amplitude. Panel (d) shows the $\left(T_{1}, 0, t\right)$ contribution subtracted by the $\left(0, T_{1}, t\right)$ contribution, which should be observed when the field of the second incident $\mathrm{THz}$ pulse is inverted.

\section{B. Nonlinear coupling}

Third-order nonlinearity in the coupling between radiation and the vibrational mode is expressed by

$$
p=q_{0} x+\frac{1}{3} q_{2} x^{3} \text {. }
$$

Then the right-hand side of Eq. (1) becomes

$$
q_{0} E \rightarrow\left(\frac{\partial}{\partial x} p\right) E=\left(q_{0}+q_{2} x^{2}\right) E
$$

The third-order polarization in this case is obtained as

$$
\begin{aligned}
P^{(3)}(t)= & \frac{q_{2}}{N^{2} q_{0}^{3}} \int_{0}^{\infty} d t_{1} \int_{0}^{\infty} d t_{2} \int_{0}^{\infty} d t_{3} r\left(t_{1}\right) r\left(t_{2}\right) r\left(t_{3}\right) \\
& \times E\left(t-t_{1}\right)\left[E\left(t-t_{1}-t_{2}\right) E\left(t-t_{1}-t_{3}\right)\right. \\
& \left.+\frac{1}{3} E\left(t-t_{2}\right) E\left(t-t_{3}\right)\right]
\end{aligned}
$$


(a)

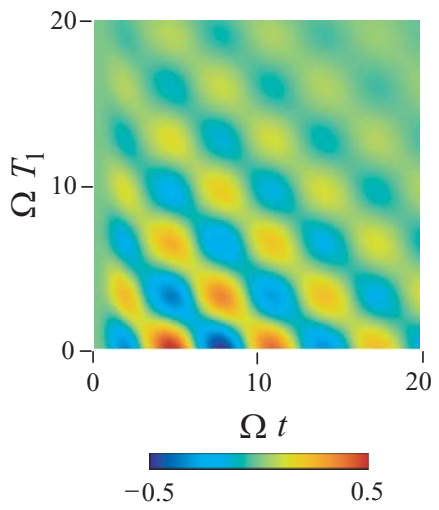

(c)

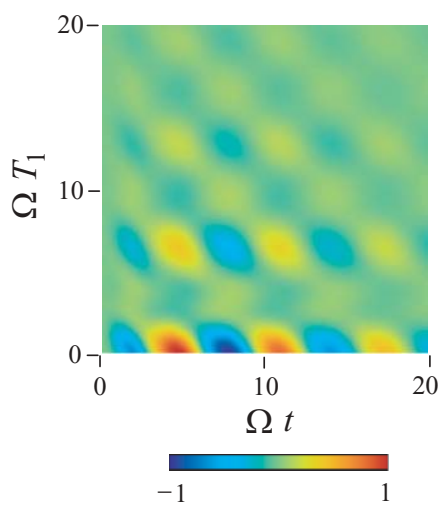

(b)

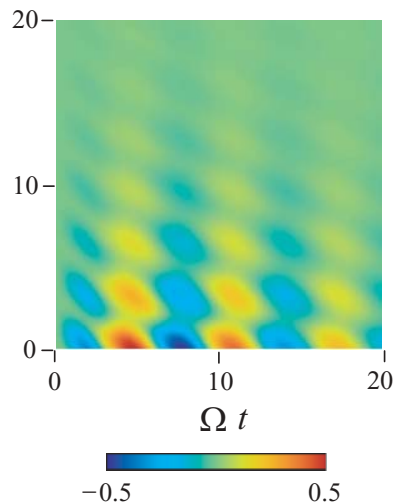

(d)

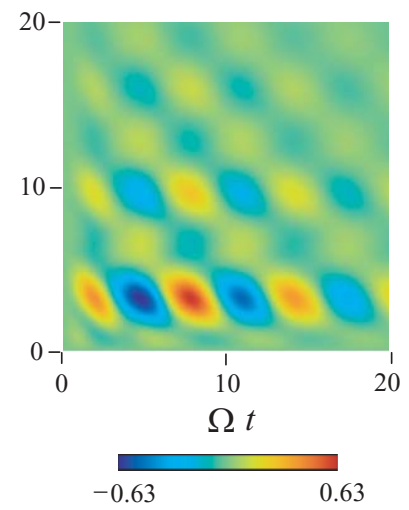

FIG. 5. Third-order THz field for a system with anharmonicity. (a) Contribution of $\left(T_{1}, 0, t\right)$ sequence. (b) Contribution of $\left(0, T_{1}, t\right)$ sequence. (c) Sum of (a) and (b). (d) Difference of (a) and (b).

By assuming delta-function excitation, the contributions of $\left(T_{1}, 0, t\right)$ and $\left(0, T_{1}, t\right)$ sequences to the third-order polarization are given by

$$
P_{\mathrm{NC}}^{(3)}\left(T_{1}, 0, t\right)=\frac{q_{2}}{N^{2} q_{0}^{3}} E_{0}^{3} r\left(t+T_{1}\right)[r(t)]^{2}
$$

and

$$
P_{\mathrm{NC}}^{(3)}\left(0, T_{1}, t\right)=\frac{q_{2}}{N^{2} q_{0}^{3}} E_{0}^{3} r(t)\left\{\left[r\left(T_{1}\right)\right]^{2}+\left[r\left(t+T_{1}\right)\right]^{2}\right\},
$$

respectively.

Third-order THz field is, accordingly, expressed as

$$
E_{\mathrm{NC}}^{(3)}\left(T_{1}, 0, t\right) \propto-\frac{d}{d t} r\left(t+T_{1}\right)[r(t)]^{2}
$$

and

$$
E_{\mathrm{NC}}^{(3)}\left(0, T_{1}, t\right) \propto-\frac{d}{d t} r(t)\left\{\left[r\left(T_{1}\right)\right]^{2}+\left[r\left(t+T_{1}\right)\right]^{2}\right\} .
$$

Numerical results for the third-order $\mathrm{THz}$ field are shown in Fig. 6.

\section{Two-level systems}

The results for a two-level (TL) system are briefly described here for comparison. In the discussion of photon echoes ${ }^{57}$ which is a third-order nonlinear optical process, and (a)

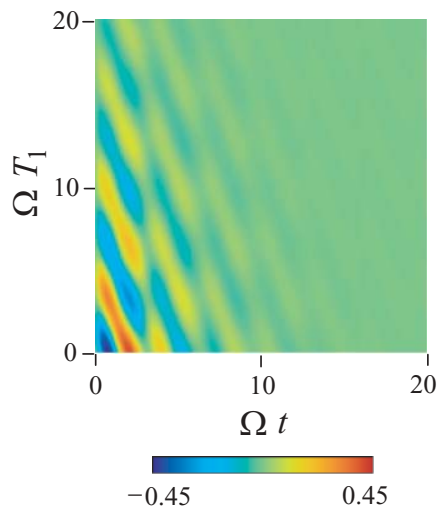

(c)

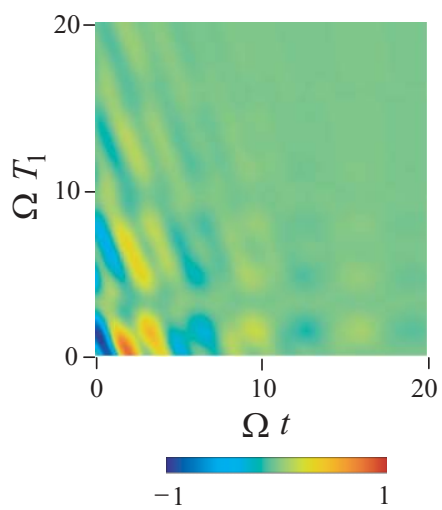

(b)

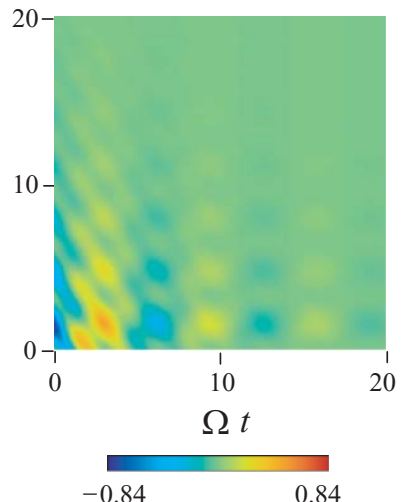

(d)

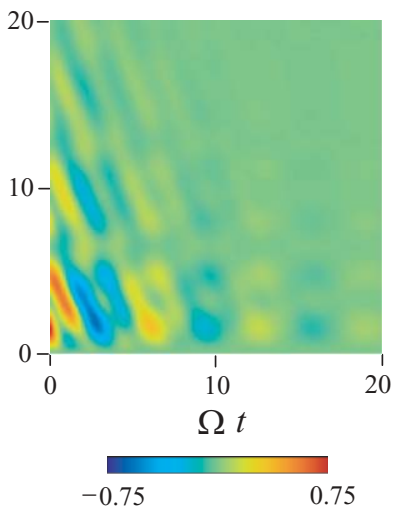

FIG. 6. Third-order $\mathrm{THz}$ field for a system with nonlinear coupling. (a) Contribution of $\left(T_{1}, 0, t\right)$ sequence. (b) Contribution of $\left(0, T_{1}, t\right)$ sequence. (c) Sum of (a) and (b). (d) Difference of (a) and (b).

Raman echoes, ${ }^{58}$ which is a seventh-order Raman process, the nonlinearity is usually assumed to originate from the twolevel nature of the systems. Two level systems have a purely quantum mechanical nature, which is in contrast to the present study, where weak nonlinearities are introduced into a classical oscillator.

Expressions for third-order polarization in two-level systems can be easily derived based on the optical Bloch equations of the system. ${ }^{59}$ For delta-function excitations, the contributions of $\left(T_{1}, 0, t\right)$ and $\left(0, T_{1}, t\right)$ sequences are expressed as

$$
\begin{aligned}
& P_{\mathrm{TL}}^{(3)}\left(T_{1}, 0, t\right) \\
& \quad=-8 N \mu\left(\frac{\mu E_{0}}{\hbar}\right)^{3} \exp \left[-\Gamma\left(T_{1}+t\right)\right] \cos \Omega T_{1} \sin \Omega t
\end{aligned}
$$

and

$$
P_{\mathrm{TL}}^{(3)}\left(0, T_{1}, t\right)=-8 N \mu\left(\frac{\mu E_{0}}{\hbar}\right)^{3} \exp \left(-T_{1} / \tau-\Gamma t\right) \sin \Omega t .
$$

Here, $\mu$ is the transition dipole moment and $\tau$ is the lifetime of the upper level population. The lifetime satisfies the condition

$$
\frac{1}{\tau} \leq 2 \Gamma .
$$


(a)

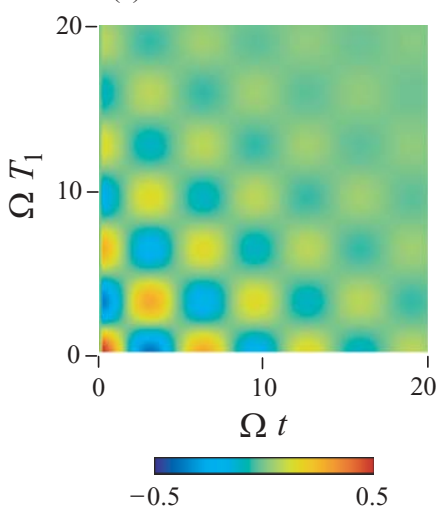

(c)

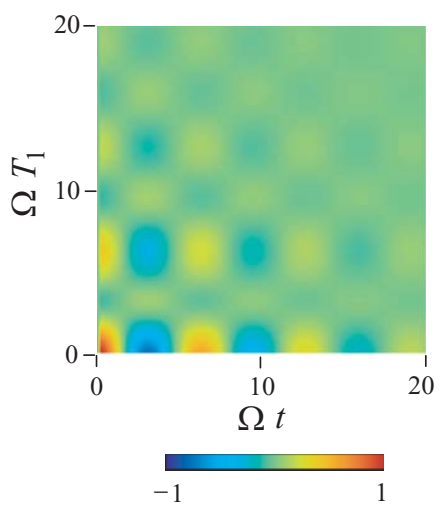

(b)

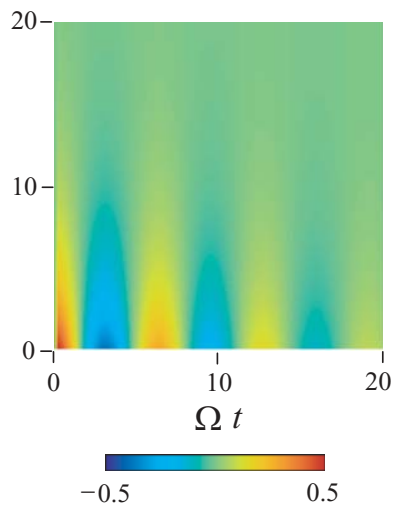

(d)

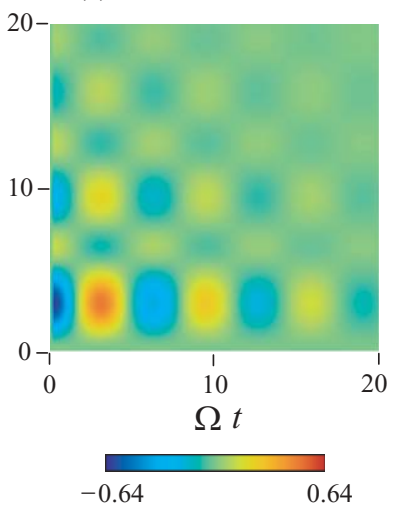

FIG. 7. Third-order $\mathrm{THz}$ field for a two-level system. (a) Contribution of $\left(T_{1}, 0, t\right)$ sequence. (b) Contribution of $\left(0, T_{1}, t\right)$ sequence. (c) Sum of (a) and (b). (d) Difference of (a) and (b).

Expressions for the third-order $\mathrm{THz}$ field are obtained by differentiating Eqs. (59) and (60) by $t$. Numerical results of the $\mathrm{THz}$ field are shown in Fig. 7, where the population lifetime is assumed as $1 / \tau=2 \Gamma$.

\section{DISCUSSION}

From the results described above, it is seen that the second-order and third-order 2D $\mathrm{THz}$ signals have specific features depending on the order and the source of nonlinearity. The second-order 2D signals shown in Fig. 2(b) for the AH case, Fig. 2(c) for the ND case, and Fig. 3(b) for the NC case exhibit characteristic features of these three origins of nonlinearity. Even when the whole 2D signal is not obtained, differences can be seen from a small subset of data. For example, on the $t=0$ line, sinusoidal oscillations are seen in the NC signal, whereas the signals vanish for the AH and ND cases. The traces on the $T_{1}=0$ line also show marked differences among them.

The third-order 2D signals depicted in Figs. 5-7 show more diverse features depending on the origin of nonlinearity and incident $\mathrm{THz}$ pulse sequence. It is seen that signals expected based on the present classical model differ significantly from those of the quantum mechanical two-level model. Consideration on the origin of nonlinearity should be important especially for the understanding of third-order signals in actual systems. There are some similarities seen between the third-order 2D profiles of the AH case and those of TL systems. This is understood by regarding the TL systems as those with an extremely large anharmonicity.

The present study is focused on discussion of the general theoretical framework for the analysis of $2 \mathrm{D} \mathrm{THz}$ spectroscopy, and expressions and numerical calculations are limited to a single mode system described by a Lorentzian model. Extensions, however, of the present model in several directions are necessary for understanding of experimental results obtained from a variety of actual systems. Some of them are easy and straightforward. Characterization of the inhomogeneity, i.e., the distribution of resonance frequency among molecules, has been a major concern of nonlinear spectroscopy, and it has been shown that fifth-order 2D Raman spectroscopy can clarify it, which is not accessible by linear spectroscopic measurements. ${ }^{19,20}$ Echo phenomena in thirdorder nonlinear processes in inhomogeneous systems are also of interest. ${ }^{58}$ Study of nonlinear coupling between different vibrational modes is another important topic, which has also been reported using fifth-order Raman spectroscopy. ${ }^{21,29}$ For this purpose Fourier transform analysis of the time-domain two-dimensional signals will be useful. ${ }^{29}$ Incorporating inhomogeneity and mode-coupling effects in multimode systems into the present model should clarify the ability of $2 \mathrm{D} \mathrm{THz}$ spectroscopy on these subjects.

Numerical calculations have been presented only for the response for delta-function incident $\mathrm{THz}$ pulses in this study. For the analysis of actual experiments, it is required to take account of a finite pulse duration since currently available $\mathrm{THz}$ pulses are usually not much shorter than the oscillation period of relevant vibrations. The line shape of the vibrational mode is Lorentzian in the present study, while those of actual modes can be different from a pure Lorentzian function. All the nonlinear responses, however, in the present study are expressed in terms of the linear response function of polarization, which is the Fourier transform of the electric susceptibility. Thus systems with any line shapes can be analyzed in the current theoretical framework. It is also possible to include nonLorentzian effects by introducing the generalized Langevin equation, ${ }^{25}$ or other type of equation of motion. Finally, molecular dynamics simulations ${ }^{38-45}$ will be necessary and powerful for understanding of dynamics in complex systems, such as liquids, in relation to the nonlinear response. Recently, Yagasaki and Saito ${ }^{60,61}$ have reported simulations on 2D infrared response of water. They simulated conventional three-pulse experiments, where the nonlinear polarization is not time-resolved. Although main features in the observed response lie in the midinfrared region, responses in the $\mathrm{THz}$ region are also included in the calculation. Comparison between 2D response described in the present paper and that of the conventional type of measurements will be helpful in understanding the dynamics of the system.

Jansen and Mukamel have shown that multitime nonlinear response functions can be expanded in terms of products of lower-order correlation functions. ${ }^{47}$ It can easily be seen that the present study is consistent with their results in the classical limit. 


\section{CONCLUSION}

A general theoretical framework of two-dimensional time-domain second-order and third-order terahertz spectroscopy has been described. The model is a classical and phenomenological one with weak nonlinearities. Three types of nonlinearity sources, anharmonicity, nonlinear coupling, and nonlinear damping, were considered. The second-order $\mathrm{THz}$ spectroscopy has an exact correspondence to fifth-order offresonance Raman spectroscopy, and it has been shown that the present treatment gives exactly the same results as of the quantum mechanical theory described by Tanimura and Mukamel $^{19}$ and Okumura and Tanimura. ${ }^{22}$ General expressions for the nonlinear signal have been obtained for a singlemode system, and numerical calculations for delta-function incident terahertz pulses were shown. For the third-order signal, two-level systems were also considered for comparison. Contributions of two types of incident pulse sequences have been studied separately in the third-order signals. Profiles of the two-dimensional $\mathrm{THz}$ signals were found to depend on the origin and order of the nonlinearity and also on the pulse sequence. The results show that the second-order and thirdorder two-dimensional signal features can clarify the nature of the system which is not accessible using linear spectroscopy.

${ }^{1}$ J. Hebling, G. Almási, and I. Z. Kozma, Opt. Express 10, 1161 (2002).

${ }^{2}$ K.-L. Yeh, M. C. Hoffmann, J. Hebling, and K. A. Nelson, Appl. Phys. Lett. 90, 171121 (2007)

${ }^{3}$ M. Jewariya, M. Nagai, and K. Tanaka, J. Opt. Soc. Am. B 26, A101 (2009).

${ }^{4}$ C. Luo, K. Reimann, M. Woerner, and T. Elsaesser, Appl. Phys. A 78, 435 (2004).

${ }^{5}$ P. Gaal, K. Reimann, M. Woerner, T. Elsaesser, R. Hey, and K. H. Ploog, Phys. Rev. Lett. 96, 187402 (2006).

${ }^{6}$ H. Wen, M. Wiczer, and A. M. Lindenberg, Phys. Rev. B 78, 125203 (2008).

${ }^{7}$ M. C. Hoffmann, J. Hebling, H. Y. H. amd K.-L. Yeh, and K. A. Nelson, Phys. Rev. B 79, 161201 (2009).

${ }^{8}$ M. C. Hoffmann, J. Hebling, H. Y. H. amd K.-L. Yeh, and K. A. Nelson, J. Opt. Soc. Am. B 26, A29 (2009).

${ }^{9}$ M. C. Hoffmann and D. Turchinovich, Appl. Phys. Lett. 96, 151110 (2010).

${ }^{10}$ M. Jewariya, M. Nagai, and K. Tanaka, "Ladder climbing on the anharmonic intermolecular potential in amino acids microcrystal with intense monocycle THz pulse," Phys. Rev. Lett. (in press).

${ }^{11}$ I. Katayama, H. Aoki, J. Takeda, H. Shimosato, M. Ashida, R. Kinjo, I. Kawayama, M. Tonouchi, M. Nagai, and K. Tanaka, Proceedings of the CLEO/QELS2010, San Jose, CA, 16-22 May 2010 (IEEE, Los Alamos, 2010), Paper No. CTuBB1.

${ }^{12}$ M. D. Levenson and S. Kano, Introduction to Nonlinear Laser Spectroscopy (Academic, New York, 1989).

${ }^{13}$ S. Mukamel, Principles of Nonlinear Optical Spectroscopy (Oxford University Press, New York, 1999).

${ }^{14}$ P. Hamm, M. Lim, and R. M. Hochstrasser, J. Phys. Chem. B 102, 6123 (1998).

${ }^{15}$ M. Cho, Two-Dimensional Optical Spectroscopy (CRC Press, London, 2009).

${ }^{16}$ K. Okumura and Y. Tanimura, Chem. Phys. Lett. 295, 298 (1998).
${ }^{17}$ Y. Suzuki and Y. Tanimura, J. Chem. Phys. 128, 164501 (2008).

${ }^{18}$ K. J. Kubarych, C. J. Milne, and R. J. D. Miller, Int. Rev. Phys. Chem. 22, 497 (2003).

${ }^{19}$ Y. Tanimura and S. Mukamel, J. Chem. Phys. 99, 9496 (1993).

${ }^{20}$ K. Okumura and Y. Tanimura, Chem. Phys. Lett. 277, 159 (1997).

${ }^{21}$ K. Okumura and Y. Tanimura, Chem. Phys. Lett. 278, 175 (1997).

${ }^{22}$ K. Okumura and Y. Tanimura, J. Chem. Phys. 107, 2267 (1997).

${ }^{23}$ V. Chernyak and S. Mukamel, J. Chem. Phys. 108, 5812 (1998).

${ }^{24}$ K. Okumura, A. Tokmakoff, and Y. Tanimura, J. Chem. Phys. 111, 492 (1999).

${ }^{25}$ J. Kim and T. Keyes, Phys. Rev. E 65, 061102 (2002).

${ }^{26}$ K. Tominaga and K. Yoshihara, J. Chem. Phys. 104, 1159 (1996).

${ }^{27}$ K. Tominaga and K. Yoshihara, J. Chem. Phys. 104, 4419 (1996).

${ }^{28}$ A. Tokmakoff and G. R. Fleming, J. Chem. Phys. 106, 2569 (1997).

${ }^{29}$ A. Tokmakoff, M. J. Lang, D. S. Larsen, G. R. Fleming, V. Chernyak, and S. Mukamel, Phys. Rev. Lett. 79, 2702 (1997).

${ }^{30}$ D. A. Blank, L. J. Kaufman, and G. R. Fleming, J. Chem. Phys. 111, 3105 (1999).

${ }^{31}$ O. Golonzka, N. Demirdöven, M. Khalil, and A. Tokmakoff, J. Chem. Phys. 113, 9893 (2000).

${ }^{32}$ D. A. Blank, L. J. Kaufman, and G. R. Fleming, J. Chem. Phys. 113, 771 (2000).

${ }^{33}$ L. J. Kaufman, D. A. Blank, and G. R. Fleming, J. Chem. Phys. 114, 2312 (2000).

${ }^{34}$ K. J. Kubarych, C. J. Milne, S. Lin, V. Astinov, and R. J. D. Miller, J. Chem. Phys. 116, 2016 (2002).

${ }^{35}$ L. J. Kaufman, J. Heo, L. D. Ziegler, and G. R. Fleming, Phys. Rev. Lett. 88, 207402 (2002).

${ }^{36}$ C. J. Milne, Y. L. Li, T. 1. C. Jansen, L. Huang, and R. J. D. Miller, J. Phys. Chem. B 110, 19867 (2006).

${ }^{37}$ Y. L. Li, L. Huang, R. J. D. Miller, T. Hasegawa, and Y. Tanimura, J. Chem. Phys. 128, 234507 (2008).

${ }^{38}$ S. Saito and I. Ohmine, J. Chem. Phys. 108, 240 (1998).

${ }^{39}$ T. 1. C. Jansen and J. G. Snijders, J. Chem. Phys. 113, 307 (2000).

${ }^{40}$ R. A. Denny and D. R. Reichman, Phys. Rev. Lett. 63, 065101 (2001).

${ }^{41}$ S. Saito and I. Ohmine, Phys. Rev. Lett. 88, 207401 (2002).

${ }^{42}$ S. Saito and I. Ohmine, J. Chem. Phys. 119, 9073 (2003).

${ }^{43}$ T. Hasegawa and Y. Tanimura, J. Chem. Phys. 125, 074512 (2006).

${ }^{44}$ S. Saito and I. Ohmine, J. Chem. Phys. 125, 084506 (2003).

${ }^{45}$ R. DeVane, B. Space, T. 1. C. Jansen, and T. Keyes, J. Chem. Phys. 125, 234501 (2006).

${ }^{46}$ C. Dellago and S. Mukamel, Phys. Rev. E 67, 035205 (2003).

${ }^{47}$ T. 1. C. Jansen and S. Mukamel, J. Chem. Phys. 119, 7979 (2003).

${ }^{48}$ S. Asaka, H. Nakatsuka, M. Fujiwara, and M. Matsuoka, Phys. Rev. A 29, 2286 (1984).

${ }^{49}$ H. Nakatsuka, A. Wakamiya, K. M. Abedin, and T. Hattori, Opt. Lett. 18, 832 (1993).

${ }^{50}$ T. Fuji, C. Jordan, T. Yoda, K. Kondo, T. Hattori, and H. Nakatsuka, Jpn. J. Appl. Phys. 39, 3429 (2000).

${ }^{51}$ T. 1. C. Jansen, J. G. Snijders, and K. Duppen, J. Phys. Chem. B 110, 19867 (2006).

${ }^{52}$ K. J. Kubarych, C. J. Milne, and R. J. D. Miller, Chem. Phys. Lett. 369, 635 (2003).

${ }^{53}$ M. Cho, D. A. Blank, J. Sung, K. Park, S. Hahn, and G. R. Fleming, J. Chem. Phys. 112, 2082 (2000).

${ }^{54}$ A. Ma and R. Stratt, J. Chem. Phys. 119, 8500 (2003).

${ }^{55}$ Y. Tanimura and S. Mukamel, J. Opt. Soc. Am. B 10, 2263 (1993).

${ }^{56}$ K. Ishii, S. Takeuchi, and T. Tahei, J. Phys. Chem. 112, 2219 (2008).

${ }^{57}$ A. Tokmakoff and M. D. Fayer, Acc. Chem. Res. 28, 437 (1995).

${ }^{58}$ R. F. Loring and S. Mukamel, J. Chem. Phys. 83, 2116 (1985).

${ }^{59}$ Y. R. Shen, The Principle of Nonlinear Optics (Wiley, New York, 1984).

${ }^{60}$ T. Yagasaki and S. Saito, J. Chem. Phys. 128, 154521 (2008).

${ }^{61}$ T. Yagasaki and S. Saito, Acc. Chem. Res. 42, 1250 (2009). 